\title{
Stable Isotope Probing of RNA combining phylogenetic microarrays and high resolution secondary ion mass spectrometry to link composition and function in microbial systems
}

\author{
X. Mayali,* P. K. Weber,* E. L. Brodie, ${ }^{*}$ S. Mabery,* P. D. Hoeprich,* I. D. Hutcheon,* and J. \\ Pett-Ridge* \\ * Lawrence Livermore National Laboratory, 7000 East Ave, Livermore CA 94550 \\ ** Lawrence Berkeley National Laboratory, 977 Berkeley Square, Berkeley CA 94704
}

A fundamental goal in microbial ecology is to understand the biogeochemical role of individual microbial taxa in their natural habitat. This rather simple concept is in actuality a complex problem because 1) most microbes remain uncultivated and 2) the majority of microbial communities are very diverse. The former makes the direct testing of isolated strains for biogeochemical activity a limited approach. The latter impacts culture-independent methods like metagenomics as many biogeochemical processes cannot be directly inferred from sequence data alone, even when assembly of complete genomes is possible.

Our approach to this problem (Chip-SIP) involves the combination of high-density phylogenetic microarrays ("chips") and stable isotope probing (SIP) to directly link identity and function. Microbial communities are incubated in the presence of stable isotope-enriched substrate(s), RNA is extracted and hybridized onto a microarray synthesized on a conductive surface (a glass slide coated with Indium Tin Oxide). The array is subsequently imaged using high resolution secondary ion mass spectrometry (SIMS) with a Cameca NanoSIMS 50 to detect isotopic enrichment. We have successfully validated this approach utilizing RNA from a single pure culture with varying degrees of isotopic enrichment using two different substrates $\left({ }^{15} \mathrm{~N}\right.$-labeled ammonium and ${ }^{13} \mathrm{C}$-labeled glucose). We show that isotopic enrichment of individual probe spots as detected by nanoSIMS is positively correlated with fluorescence as detected by a traditional microarray scanner. This allows the relationship between hybridization efficiency and relative isotopic enrichment to be determined. Further, we have successfully detected ${ }^{15} \mathrm{~N}$ enrichment in an estuarine bacterial community following incubation with ${ }^{15} \mathrm{~N}-\mathrm{NH}_{4}$, demonstrating the utility of the method in mixed natural communities. Current efforts are aimed at elucidating the major players in the incorporation of organic carbon in estuarine microbial communities. It is currently unknown if members of heterotrophic communities in these eutrophic systems are generalists or instead specialize in the incorporation of specific substrates such as amino acids, starch, and fatty acids. We are employing the Chip-SIP approach to determine which taxa incorporate carbon from which substrates to gain a better understanding of organic matter processing in these coastal environments. 